\title{
Impact of Fatigue on Quality of Life among COVID-19 Survivors
}

\author{
Priya $\mathbf{S}^{*}$, Abhilash P.V ${ }^{* *}$, Gloria Mohan Naik ${ }^{* * *}$ \\ *Associate Professor, (MPT) ${ }^{* *}$ Associate Professor, (MPT), ${ }^{* * *}$ Post Graduate Student, \\ Laxmi Memorial College of Physiotherapy, Rajiv Gandhi University of Health Sciences, Karnataka, India \\ Corresponding Author: Gloria Mohan Naik
}

\begin{abstract}
Background: COVID-19 is a truly multisystem disease, with common extra-respiratory complications affecting the cardiac, renal, gastrointestinal, nervous, endocrine and musculoskeletal systems. Fatigue is a common symptom in those presenting with symptomatic COVID-19 infection. However, it is unknown if COVID-19 results in persistent fatigue in those recovered from acute infection. There is currently very limited information on the nature and occurrence of post-COVID-19 symptoms after hospital discharge and its impact on quality of life. This study aims to assess those recovering from COVID-19 for symptoms of fatigue and its impact on quality of life, thus identifying a group worthy of further study and early intervention.
\end{abstract}

Objective: To determine the impact of fatigue on quality of life among COVID-19 survivors.

Methods: 99 patients exposed with covid-19 virus and tested negative before 6 weeks was followed up and assessed for severity of fatigue and its impact on quality of life. Participants were asked on a telephone to choose an appropriate option for each question in relation to current situation from the Chalder fatigue scale and COV19 quality of life questionnaire in COVID 19 survivors.

Result: Participants with mean age of $32.6 \pm 11.28$ years and majority between 23-27 years were included. In this present study males were affected more than females. Total 67 participants experienced fatigue out of which 47 had an impact on their quality of life. A chi square test of independence was performed to determine the impact of fatigue on quality of life in COVID19 survivors. Impact of fatigue on quality of life was statistically significant, $\left[\mathrm{X}^{2}(1\right.$,
$\mathrm{N}=99)=6.14, \mathrm{p}=0.013$ ] with small effect size of 0.249 .

Conclusion: Present study concludes that, there was a significant impact of fatigue on the quality of life. Hence, the fatigue and its associated symptoms need to be monitored and early intervention to be planned in post COVID19 patients. Home exercises and cognitive therapy can be taught to improve the capacity and quality of life.

Keywords: COVID-19, Fatigue Level, Chalder Fatigue Scale, quality of life.

\section{INTRODUCTION}

COVID-19 is a viral infection caused by SARS-CoV-2 that primarily targets the respiratory system, with initial symptoms often including shortness of breath and fever ${ }^{1}$. As of 28 May 2020, there are over 5.5 million confirmed cases of people who have contracted COVID-19 globally and over 353,000 have expired ${ }^{2}$. For coronavirus disease COVID-19 patients, overcoming the acute symptoms of the disease may only be the beginning of a long and challenging path to recovery ${ }^{3}$. It has been shown that after viral infections (e.g., SARS-1), patients often sustain functional limitations over a long period after discharge from the hospital ${ }^{3}$. In many cases, physical, cognitive, and psychological impairments persist for multiple years ${ }^{4}$. Similarly, as COVID-19 research progresses, it has become increasingly apparent that a high proportion of patients experience persistent symptoms, such as fatigue ${ }^{5}$. 
A commonly reported symptom of COVID-19 is fatigue, and anecdotal evidence suggests that some people continue to experience severe levels of prolonged fatigue as they recover from this infection ${ }^{6}$. Fatigue is recognized as one of the most common presenting complaints in individuals infected with SARS-CoV-2, the cause of the current COVID-19 pandemic ${ }^{7}$. In early reports on the clinical characteristics of those infected, fatigue was listed as a presenting complaint in $44 \%-$ $69.6 \%^{7}$. Further studies were followed by meta-analyses, with $34-46 \%$ of those infected presenting with fatigue ${ }^{7}$. The levels of both physical and psychological fatigue seen post-COVID are higher than those of the general population, but do not reach the levels of those seen in chronic fatigue syndrome $^{7}$. Whilst the presenting features of SARS-CoV-2 infection have been wellcharacterized, with symptoms ranging from mild taste and smell disturbance to dyspnea and respiratory failure, the medium and long-term consequences of SARS-CoV-2 infection remain unexplored ${ }^{8}$. In particular, concern has been raised that SARS-CoV-2 has the potential to trigger a post-viral fatigue syndrome ${ }^{9 .}$ This is not surprising, as post-infectious fatigue has been widely observed across a variety of other viral and non-viral infections ${ }^{10}$. Patients who survive may be susceptible to developing poor health related quality of life and persistent symptom after discharge ${ }^{11}$.

Evidence from previous large-scale health outbreaks suggests that this type of event has a tremendous impact not only on physical health, but also on mental health and quality of life in general (Sim \& Chua) 12. 'The COV-19- Impact on Quality of Life(COV19-QoL)', a scale aiming at capturing the effect of COVID-19 on people's quality of life ${ }^{13}$. Furthermore to date, the impact of fatigue on quality of life (QOL), a widely used comprehensive outcome in COVID19 survivors is unknown.

Therefore the primary aim is to establish whether patients recovering from
SARS-CoV-2 infection remained fatigued after their physical recovery, and to investigate whether there was any impact of fatigue on quality of life among COVID19 survivors. Present study hypothesized that the prevalence of fatigue among COVID19 survivors would be higher. COVID19 survivors with fatigue might have a lower quality of life than those of non-fatigued.

\section{METHODOLOGY \\ Design and setting}

A cross section based survey was conducted in a tertiary care hospital and was approved by the Institutional Ethical Committee.

\section{Participants}

99 COVID 19 survivors, more than 6 weeks of testing negative by RT PCR at tertiary care hospital were included in this study. Patients under 18 years or with no contact details available, or with dementia, learning disability, cognitive or communication impairments and those who refused to respond were excluded from the study.

\section{Procedure}

Each participant took part in only one session through telephone. A brief introduction about the survey procedure was explained to all the subjects. Participants were recruited on basis of the inclusion and exclusion criteria and an initial examination included demographic data such as Name, Age, Gender, Contact no, Date of COVID19 positive, Date of discharge, Occupation and Associated co-morbidities. Participants were asked to choose an appropriate option for each question in relation to current situation from the Chalder fatigue scale and COV19 quality of life questionnaire in COVID 19 survivors.

\section{Outcome measures}

\section{Fatigue}

Fatigue was assessed using the validated Chalder Fatigue Scale (CFQ-11). Briefly, participants are asked to answer these questions with particular reference to 
the past month in comparison to their preCOVID-19 baseline, the CFQ also allows the differentiation of "cases" $\mathrm{v} / \mathrm{s}$ "noncases" where scores 0 and 1 ("Better than usual"/"No worse than usual") are scored a zero and scores 2 and 3 ("Worse than usual"/"Much worse than usual") are scored a 1 (bimodal scoring). The sum of all 11 binary scores was calculated and those with a total score of 4 or greater considered meeting the criteria for fatigue. This latter method for "caseness" is validated and closely resembles other fatigue questionnaires. For the current study, we computed: (i) case-status (fatigue vs. nonfatigued) using the bimodal scoring method $^{14}$.

\section{Quality of life}

The COV19-Quality of life is a 6item scale covering main areas of quality of life in relation to mental health. Selection of items was guided by the idea to cover the main areas that are thought to be mainly impacted from a large-scale public health outbreak: quality of life and mental health symptoms (their relevance was highlighted by Zhang \& Ma) ${ }^{15}$ as well as personal safety its importance was underlined by Singer et al) ${ }^{16}$. All items included a 5-point Likert scale (1-"totally disagree" to 5"completely agree") and assess a period of last 7 days. Total scores are calculated by averaging the scores on all the items. A higher score indicates greater perceived impact of the pandemic on one's quality of life ${ }^{13}$.

\section{Statistical analysis}

SPSS ver.20 was used to analyse data and descriptive statistics was used for demographic data such as age. Chi square test was used to find the impact of fatigue on quality of life in COVID19 survivors.

\section{RESULT}

The total number of 99 COVID19 survivors aged 18-55 years, tested negative by RT-PCR 6 weeks back with the minimum age of 18 to maximum of 54 with mean of 32.6 and standard deviation of 11.28 were included in this study. Descriptive statistics was used to find out the age, fatigue and quality of life. Majority were seen in 23-27 year age group. $\mathrm{P}<0.005$ was considered statistically significant.

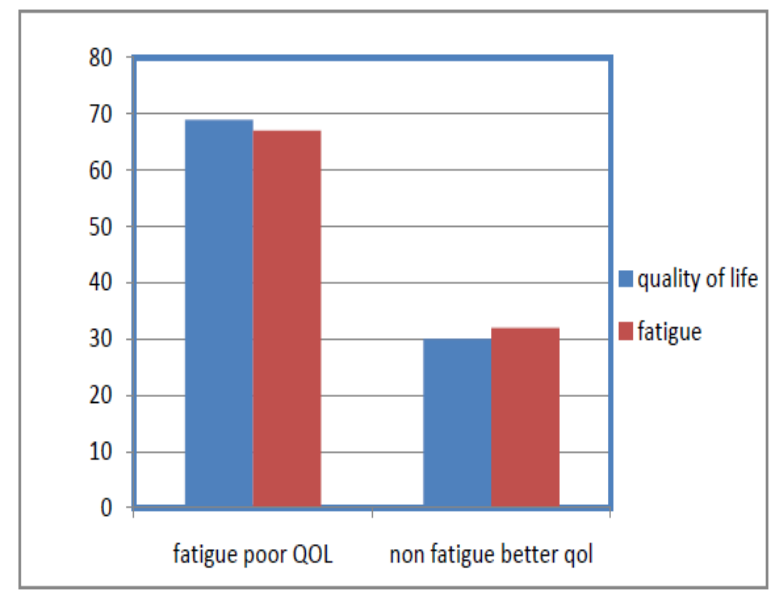

Fig.1 Descriptive statistics of fatigue and quality of life

Table 1: Chi square test of independence to find impact of fatigue on quality of life

\begin{tabular}{|c|c|c|c|}
\hline & Chi square value & df & p value \\
\hline Pearson chi square & 6.148 & 1 & 0.013 \\
\hline
\end{tabular}

A Chi square test of independence was performed to determine the impact of fatigue on quality of life among COVID19 survivors. Impact of fatigue on quality of life was statistically significant, $\left[\mathrm{X}^{2}(1\right.$, $\mathrm{N}=99)=6.14, \mathrm{p}=0.013]$ with small effect size of 0.249 .

\section{DISCUSSION}

Present study was done to determine the impact of fatigue on quality of life among COVID19 survivors. The COVID19 pandemic is a significant psychological and physiological stressor for individuals and healthcare system. Shechter A. et al. studied the psychological distress, coping behaviors, and the preferences for support among health workers during COVID19 pandemic and revealed that healthcare workers are experiencing enormous distress with large proportions screening positive for acute stress, depressive symptom and sleep disturbances ${ }^{17}$. Chaix $\mathrm{B}$ et al. studied psychological distress during the COVID19 pandemic in France: a national assessment of at-risk population and revealed that 
prevalence of psychological distress is high in at-risk (asthma, breast cancer, depression and migraine) patients ${ }^{18}$. The present study revealed that $67 \%$ of COVID 19 survivors reported fatigue. El Sayed S. et al ${ }^{19}$ studied post COVID 19 fatigue and anhedonia and revealed that there is high prevalence of fatigue in post COVID 19 with the mean of, 40.81, this is in agreement with Huang, et al. $^{20}$ who noted that most patients in post COVID 19 period developed muscle ache, arthralgia, weakness, fatigue or myalgia. In this study $69 \%$ of COVID 19 survivors reported poor quality of life which supports this study. Todt $\mathrm{B}^{21}$ et al. studied clinical outcomes and quality of life of COVID 19 survivors and noted that there is impairment in quality of life and mental health and possibly resulting on increased long-term demand to healthcare system.

Present study found that $47.4 \%$ of COVID 19 survivors reported fatigue and had an effect on quality of life during COVID 19 outbreak. Zou $S$ et $a^{22}$ studied fatigue and its association with quality of life among clinically stable older psychiatric patients during COVID 19 outbreak and noted that prevalence of fatigue was $47.1 \%$, indicating that it is common among clinically stable older psychiatric patients during COVID 19 pandemic. The study found that patients with fatigue had lower quality of life and it may be due to various predisposing factors (depressive or anxiety disorders, limited access to psychiatric service, sedentary lifestyle, lack of outdoor activities and physical exercise, fear of transmission and chronic physical illness) which were in support with this study. There is a significant burden of fatigue at follow up after 6 weeks, with more half of the patient reporting fatigue. This has several implications. Firstly, more than $50 \%$ of the participants do not feel back to full health, despite being medically deemed recovered from their primary illness. Secondly, there might be an impact of this fatigue on quality of life. This is of particular concern, since it is recommended that post-viral infection return to work should take place after four weeks to prevent deconditioning. Vink $M$ et $\mathrm{al}^{23}$ studied work rehabilitation and medical retirement for myalgic encephalomyelitis/ chronic fatigue syndrome patients and revealed that successful return to work will require ongoing input from occupational health departments and employers. Koopmans $\mathrm{P}$ et $\mathrm{al}^{24}$ studied return to work following sickness absence due to infectious mononucleosis and reported that return to work should be 4 weeks after the onset of infection and it may be due to poor physical functioning causing prolonged symptom. The levels of both physical and psychological fatigue in seen in postCOVID are higher, but do not reach the levels of those seen in chronic fatigue syndrome. Morgul E et $\mathrm{al}^{25}$ studied COVID19 pandemic and psychological fatigue and found individuals felt physically and mentally exhausted, feeling tired quickly, lack of energy, inability to start and perform everyday activities, lack of desire to do things and concentrated on work. Other reason might be lack of outdoor activities and physical exercise due to quarantine measures during the COVID 19 pandemic indirectly impairing the overall quality of life. Fatigue might be associated with sleep disturbances, reduced energy and motivation, fear of recurrent infection, all of which could negatively affect daily functioning, increases physical and mental distress and eventually lower quality of life.

These study findings suggest that all patients diagnosed with SARS-CoV-2 will require screening for fatigue and quality of life. It is also believed that, the effect of fatigue on quality of life and return to work is profound, especially when the number of patients that will be affected and its potential impact on individuals, employers and governments. Management of fatigue states requires multi-disciplinary input and will not be appropriately addressed if follow-up is by treating medical physicians alone. Various interventions, including graded exercise and cognitive behavioral therapy ${ }^{26,27}$, are needed to manage CFS and may be relevant to post infectious fatigue. 
This study has shown that, there is significant impact of fatigue on quality of life, commonly from 1.5-6 months after COVID-19 recovery and it requires monitoring and management.

\section{Limitation}

- Face to face interview is not possible hence selection bias which may affect generalization of study.

- Non response rate is probably high.

- Responses to some of the questions, such as symptoms during COVID-19, may have been influenced by recall bias.

\section{CONCLUSION}

This study concludes that, there was a predominant impact of fatigue on quality of life among COVID 19 survivors on physical and psychological aspects. Priority should be given to examine the prevalence of fatigue and long-term monitoring of its related symptoms following COVID19 infection and to explore low cost and convenient techniques to treat post-viral fatigue, to resolve the symptoms and improve quality of life in COVID19 survivors.

Acknowledgement: I would like to thank the participants who took part in the study.

Conflict of Interest: Authors reports no conflict of interest.

\section{Source of Funding: None}

\section{Ethical Approval: Approved}

\section{REFERENCES}

1. Rothan HA, Byrareddy SN. The epidemiology and pathogenesis of coronavirus disease (COVID-19) outbreak. J Autoimmun. 2020; 109:102433.

2. Islam M, Cotler J, Jason L. Post-viral fatigue and COVID-19: lessons from past epidemics. Fatigue2020;8 (2):61-69.

3. Rudroff T, Fietsam AC, Deters JR, Bryant AD, Kamholz J. Post-COVID-19 Fatigue: Potential Contributing Factors. Brain Sci. 2020; 10(12):1012.
4. Lam MH, Wing YK, Yu MW, Leung CM, Ma RC, Kong AP, So WY, Fong, SY, Lam, SP. Mental morbidities and chronic fatigue in severe acute respiratory syndrome survivors: Long-term follow-up. Arch. Int. Med. 2009, 169(22)2142-2147

5. Goertz YM, Van Herck M, Delbressine JM, Vaes AW, Meys, R.; Machado FV, HoubenWilke S, Burtin C, Posthuma R, Franssen, FM, van Loon N. Persistent symptoms 3 months after a SARS-CoV-2 infection: The post-COVID-19 syndrome? ERJ Open Res. 2020; 6(4).

6. Del Rio C, Malani PN. COVID19 -New insights on a rapidly changing epidemic. Jama. 2020; 323(14):1339-1340.

7. Townsend L, Dyer AH, Jones K, Dunne J, Mooney A, Gaffney F, O'Connor L, Leavy D, O'Brien K, Dowds J, Sugrue JA: Persistent fatigue following SARS-CoV-2 infection is common and independent of severity of initial infection. Plos one. 2020;15(11):e024078

8. Huang C, Wang Y, Li X, Ren L, Zhao J, Hu $\mathrm{Y}$, et al. Clinical features of patients infected with 2019 novel coronavirus in Wuhan, China. The lancet. 2020; 395(10223):497-506.

9. Wilson C. Concern coronavirus may trigger post-viral fatigue syndromes. New Scientist 2020; 246(3278): 10

10. Hickie I, Davenport T, Wakefield D, et al. Post-infective and chronic fatigue syndromes precipitated by viral and nonviral pathogens: prospective cohort study. Bmj. 2006; 333(7568):575.

11. Taboada M, Moreno E, Cariñena A, Rey T, Pita-Romero R, Leal S et al. Quality of life, functional status, and persistent symptoms after intensive care of COVID-19 patients. Br J Anaesth. 2021; 126(3):e110-e113.

12. Sim, K. \& Chua, HC. The psychological impact of SARS: a matter of heart and mind. Can. Med. Assoc J, 2004; 170(5), 811-812.

13. Repišti S, Jovanović N, Kuzman M, Medved S, Jerotić S, Ribić E et al. How to measure the impact of the COVID-19 pandemic on quality of life: COV19-QoLthe development, reliability and validity of a new scale. Global Psychiatry. 2020; 1(0).

14. Jackson C. The Chalder Fatigue Scale (CFQ 11). Occup. Med. 2015; 65(1):86-86.

15. Zhang, Y. \& Ma, ZF. Impact of the COVID19 pandemic on mental health and quality of 
life among local residents in Liaoning Province, China: A cross-sectional study. Int. J. Environ. Res. Public Health. 2020;(7)2381-2391.

16. Singer, PA., Benater SR., Bernstein M, Daar, AS, Dickens BM., MacRae SK, Upshur RE, Wright L, Shaul RZ. Ethics and SARS: lessons from Toronto. Bmj. 2003; 327(7427)1342-1344

17. Shechter A, Diaz F, Moise N, Anstey De et al. Psychological distress, coping behaviors, and preferences for support among New York healthcare workers during the COVID19 pandemic. Gen. Hosp. Psychiatry. 2020; 66(8)1-8.

18. Chaix B, Delamon G, Guillemasse A, Brouard B, Bibault JE. Psycholocical distress during the COVID19 pandemic in France: a national assessment of at-risk populations. Gen. Psychiatry.2020;33(6):

19. El Sayed S, Shokry D, Gomaa S. PostCOVID-19 fatigue and anhedonia: A crosssectional study and their correlation to postrecovery period. Neuropsychopharmacol Rep. 2021; 41(1): 50-55.

20. Haung C, Wang Y, Li X, Ren L, Zhao J, Hu Y. Cinical features of patients infected with 2019 novel coronavirus in Wuhan, China. Lancet. 2020; 395; 497-506.

21. Todt B, Szlejf C, Duim E, Linhares A, Kogiso D, Varela G et al. Clinical outcomes and quality of life of COVID19 survivors: A follow-up of 3 months post hospital discharge. Resp Med. 2021; 184: 106453.

22. Zou S, Liu Z, Yan X, Wang H, Li Y, Xu X et al. Prevalence and correlates of fatigue and its association with quality of life among clinically stable older psychiatric patients during the COVID19 outbreak: a cross-sectional study. Glob and Health 2020; 16(1).

23. Vink M, Vink-Niese F. Work Rehabilitation and Medical Retirement for Myalgic Encephalomyelitis/Chronic Fatigue Syndrome Patients. A Review and Appraisal of Diagnostic Strategies. Diagnostics. 2019; 9(4):124.

24. Koopmans P, Bakhtali R, Katan A, Groothoff J, Roelen C. Return to work following sickness absence due to infectious mononucleosis. Occup Med. 2010; 60(4):249-54.

25. Morgul E, Bener A, Atak M, Akyel S, Aktas S. et al. COVID19 pandemic and psychological fatigue in Turkey. Int. J. of soci psyc. 2020

26. White PD, Goldsmith KA, Johnson AL, Potts L, Walwyn R, DeCesare JC, et al. Comparison of adaptive pacing therapy, cognitive behaviour therapy, graded exercise therapy, and specialist medical care for chronic fatigue syndrome (PACE): a randomised trial. The Lancet. 2011; 377 (9768):823-36.

27. Galeoto G, Sansoni J, Valenti D, Mollica R, Valente D, Parente M, et al. The effect of physiotherapy on fatigue and physical functioning in chronic fatigue syndrome patients: a systematic review. La Clinica Terapeutica. 2018; 169(4):e184-e8

How to cite this article: Priya S, Abhilash P.V, Naik GM. Impact of fatigue on quality of life among COVID-19 survivors. International Journal of Science \& Healthcare Research. 2021; 6(3): 327-332. DOI: https://doi.org/ 10.52403/ijshr.20210756 